\title{
Risk stratification of pulmonary embolism: clinical evaluation, biomarkers or both?
}

\author{
Guy Meyer ${ }^{1,2,3,4,5}$, Benjamin Planquette ${ }^{1,5,6}$ and Olivier Sanchez $z^{1,2,5,6}$
}

Affiliations: 'Service de Pneumologie, Hôpital Européen Georges Pompidou, APHP, Paris, France. ${ }^{2}$ Université Paris Descartes, Sorbonne Paris Cité, Paris, France. ${ }^{3}$ INSERM UMRS 970, Paris, France. ${ }^{4} \mathrm{CIC} 1418$, Paris, France. ${ }^{5}$ GIRC Thrombose, Saint-Etienne, France. ${ }^{6}$ INSERM UMR-S 1140, Paris, France.

Correspondence: Guy Meyer, Service de Pneumologie, Hôpital Européen Georges Pompidou, 20 rue Leblanc, 75015 Paris, France. E-mail: guy.meyerdegp.aphp.fr

@ERSpublications

A predicting rule based on copeptin may be better than ESC criteria for risk stratification of pulmonary embolism http://ow.ly/T4APZ

The concept of risk-stratification is one of the cornerstones of the recent guidelines on pulmonary embolism (PE), which were issued by the European Society of Cardiology (ESC) and endorsed by the European Respiratory Society [1]. According to these guidelines, patients with PE are divided in three groups with increasing risk of death or adverse outcome following a three-step process that is based on clinical data, assessment of anatomical right ventricular dysfunction on echocardiography or spiral computed tomography (CT), and of myocardial injury by dosage of cardiac biomarkers [1]. The first step is based on the measurement of blood pressure to determine whether cardiogenic shock is present, or not. For the vast majority of patients with acute PE who present with normal blood pressure, the second step consists of a simple clinical evaluation, summarised in the Pulmonary Embolism Severity Index (PESI) or its simplified version (sPESI) [2,3]. These scores are able to select low-risk patients, defined as PESI class I or II or sPESI $=0$ who have a mortality risk $<3 \%$ at 1 month. Cohort studies and one randomised clinical trial have confirmed that low-risk patients can safely be treated at home $[4,5]$. Implementing PESI enables the identification of $\sim 45 \%$ of patients with acute PE as low-risk patients and reduces the length of hospitalisation without significantly increasing the risk of death, recurrent PE or major bleeding $[4,6]$. Patients with normal blood pressure, but higher values of PESI or sPESI, have a mortality risk of $\sim 5-7 \%$ and should be treated at hospital according to ESC guidelines $[1,7,8]$. The goal of further risk stratification is to select the patients with the highest risk of an adverse event among those with normal blood pressure. This can be done by the combination of cardiac biomarkers dosing and/or the search for right ventricular dysfunction (RVD) by echocardiography or spiral CT. Several cohort studies strongly suggest that clinical variables, RVD and biomarkers have independent and complementary prognostic values in normotensive patients with acute PE [8-10].

In this issue of the European Respiratory Journal, HellenKamp et al. [11] report on a quite different approach. Instead of combining clinical variables or clinical rules with biomarkers and echocardiographic data, which may be both time consuming and difficult to implement in all hospitals, these authors propose a risk-stratification process entirely based on biomarkers (ref). In this study, 268 normotensive patients with acute PE were stratified in three groups of increasing severity according to the levels of three different biomarkers, copeptin, high-sensitivity troponin $\mathrm{T}$ (hsTnT) and N-terminal pro-brain natriuretic peptide (NT-proBNP). None of the 141 patients (52.6\%) with hsTnT $<14 \mathrm{pg} \cdot \mathrm{mL}^{-1}$ or NT-proBNP $<600 \mathrm{pg} \cdot \mathrm{mL}^{-1}$ had an adverse outcome. Copeptin was then used to further stratify patients with elevated hsTnT and NT-proBNP. Copeptin was $<24 \mathrm{pmol} \cdot \mathrm{L}^{-1}$ in 71 patients $(26.6 \%$ of the cohort) and four of these patients $(5.6 \%)$ experienced an adverse outcome. Finally, copeptin was $>24 \mathrm{pmol} \cdot \mathrm{L}^{-1}$ in 55 patients (20.6\% of the cohort) and 11 of these patients (20\%) experienced an adverse outcome.

Received: Sept 202015 | Accepted: Sept 222015

Conflict of interest: Disclosures can be found alongside the online version of this article at erj.ersjournals.com

Copyright @ERS 2015 
How do these results compare with previously described risk-assessment scores in normotensive patients with PE?

In the study reported by HellenKamp et al. [11] the results of the biomarker-based approach compared favourably with those of more traditional approaches combining clinical data and biomarkers. In fact, the positive predictive value (95\% confidence interval) for the adverse 30-day outcome of the copeptin-based score, the ESC score and the Bova score were 0.20 (0.12-0.32), $0.12(0.06-0.20)$ and $0.11(0.05-0.24)$, respectively [11]. Conversely, the three scores obtained high and comparable levels of negative predictive value, but the biomarker-based approach classified a larger proportion of patients as low risk (52\%) than the ESC score, (17.5\%). These comparisons should be interpreted with caution for at least two reasons. First, a score usually works better in the population on which it has been derived and secondly, the biomarkers were measured in all patients as per protocol, which is not required by ESC guidelines that advocate biomarkers measurement only in patients with high values of PESI or sPESI [1]. Previously described risk assessment models were already able to select normotensive patients with a risk of major adverse event. A sPESI $\geqslant 1$, with a brain natriuretic peptide $>100 \mathrm{pg} \cdot \mathrm{mL}^{-1}$ and a troponin $>0.05 \mathrm{ng} \cdot \mathrm{mL}^{-1}$ was associated with a predicted risk of 30-day complicated course of $19.2 \%$ in normotensive patients [10]. In the study by Bova et al. [12] patients with blood pressure $>90 \mathrm{mmHg}$ who have a score $\geqslant 4$ based on systolic blood pressure, RVD and troponin had a risk of PE-related complication of $29.2 \%$ at 30 days. The FAST score, based on heart-type fatty acid-binding protein, syncope, and tachycardia also demonstrated a $22 \%$ positive predictive value in the derivation cohort of normotensive patients with PE [13]. In the subset of normotensive patients included in the PREP (Prognostic Factors for Pulmonary Embolism) study, the combination of sPESI, RVD and biomarkers had a $21.2 \%$ positive predictive value [10].

\section{What is the clinical significance of a $20 \%$ positive predictive value?}

All in all, these results strongly suggest that combination of clinical data, biomarkers and evidence of RVD on imaging or the combination of several biomarkers are able to select a high-risk group of patients within those presenting with normal blood pressure. A rule with a $20 \%$ positive predictive value can be considered as a poor predictor of outcome. However, this means that about one in five such patients who present with normal blood pressure but have elevated biomarkers, will experience major adverse events including: PE-related death, the need for intravenous catecholamine administration, mechanical ventilation and/or cardiopulmonary resuscitation, despite anticoagulant treatment.

For the time being, guidelines recommend close monitoring of these "high-intermediate risk" patients with the hope that early detection of haemodynamic collapse/shock should ensure a prompt and effective treatment allowing for a reduction in the risk of death [1]. Although this recommendation makes sense and is following common sense, an intervention aimed at preventing haemodynamic decompensation would be welcome. Recent data suggest that thrombolysis is able to reduce significantly the rate of haemodynamic decompensation or death by approximately $50 \%$, but this was obtained in a group of patients with an absolute rate of events of about $6 \%$ and at the cost of a significant increase of major and intracranial bleedings [14]. If the same relative reduction in the risk of major PE-related outcomes (i.e. haemodynamic decompensation, death due to PE or recurrent PE) is achieved in a group of patients with a risk of events of approximately $20 \%$ using a fibrinolytic regimen with a lower risk of bleeding, this would change the risk to benefit ratio of reperfusion therapy in this context.

\section{What could be the next steps?}

We now have several rules that have been derived to select patients with a significant risk of PE-related major complications of $\sim 20 \%$, but these models have still a low level of validation. Some were derived in single centres with a high expertise questioning their generalisability, the clinical variables and cardiac biomarkers vary from one study to another and most predictive models lack external validation [8-10, 12]. This is crucial because usually a rule works much better in the population where it was derived than in other settings, questioning the reliability of the $20 \%$ positive predictive value reported by the different rules. Finally, no prospective interventional study has, as of yet, ever proven that the implementation of a risk-stratification process results in the improvement in patient-oriented outcomes.

It is now probably time to validate externally these different rules in a large multicentre cohort using a blinded adjudication of outcomes rather than searching again and again for the best combination of predictors. A multicentre cohort allowing a face-to-face comparison between mixed clinical and biological approaches and exclusive biomarkers approach in different populations and settings would be helpful for achieving this goal. If this study confirms that one or several rules are able to select patients with a $20 \%$ risk of major PE-related major complications, the next step could be to redo a trial assessing a safer reperfusion therapy regimen in these patients. 


\section{References}

1 Konstantinides SV, Torbicki A, Agnelli G, et al. 2014 ESC guidelines on the diagnosis and management of acute pulmonary embolism. Eur Heart J 2014; 35: 3033-3069.

2 Aujesky D, Obrosky DS, Stone RA, et al. Derivation and validation of a prognostic model for pulmonary embolism. Am J Respir Crit Care Med 2005; 172: 1041-1046.

3 Jiménez D, Aujesky D, Moores L, et al. Simplification of the pulmonary embolism severity index for prognostication in patients with acute symptomatic pulmonary embolism. Arch Intern Med 2010; 170: 1383-1389.

4 Aujesky D, Roy PM, Verschuren F, et al. Outpatient versus inpatient treatment for patients with acute pulmonary embolism: an international, open-label, randomised, non-inferiority trial. Lancet 2011; 378: 41-48.

5 Zondag W, Kooiman J, Klok FA, et al. Outpatient versus inpatient treatment in patients with pulmonary embolism: a meta-analysis. Eur Respir J 2013; 42: 134-144.

6 Kohn CG, Mearns ES, Parker MW, et al. Prognostic accuracy of clinical prediction rules for early post-pulmonary embolism all-cause mortality: a bivariate meta-analysis. Chest 2014; 147: 1043-1062.

7 Zondag W, den Exter PL, Crobach MJ, et al. Comparison of two methods for selection of out of hospital treatment in patients with acute pulmonary embolism. Thromb Haemost 2013; 109: 47-52.

8 Lankeit M, Jiménez D, Kostrubiec M, et al. Validation of N-terminal pro-brain natriuretic peptide cut-off values for risk stratification of pulmonary embolism. Eur Respir J 2014; 43: 1669-1677.

9 Sanchez O, Trinquart L, Caille V, et al. Prognostic factors for pulmonary embolism: the PREP study, a prospective multicenter cohort study. Am J Respir Crit Care Med 2010; 181: 168-173.

10 Jiménez D, Kopecna D, Tapson V, et al. Derivation and validation of multimarker prognostication for normotensive patients with acute symptomatic pulmonary embolism. Am J Respir Crit Care Med 2014; 189: $718-726$.

11 Hellenkamp K, Schwung J, Rossmann H, et al. Risk stratification of normotensive pulmonary embolism: prognostic impact of copeptin. Eur Respir J 2015; 46: 1701-1710.

12 Bova C, Sanchez O, Prandoni P, et al. Identification of intermediate-risk patients with acute symptomatic pulmonary embolism. Eur Respir J 2014; 44: 694-703.

13 Dellas C, Tschepe M, Seeber V, et al. A novel H-FABP assay and a fast prognostic score for risk assessment of normotensive pulmonary embolism. Thromb Haemost 2014; 111: 996-1003.

14 Meyer G, Vicaut E, Danays T, et al. Fibrinolysis for patients with intermediate-risk pulmonary embolism. $N$ Engl $J$ Med 2014; 370: 1402-1411. 